\title{
GmBZL3 acts as a major BR signaling regulator through crosstalk with multiple pathways in Glycine max
}

Li Song ${ }^{1,2+}$, Wei Chen ${ }^{2 \dagger}$, Biao Wang ${ }^{3}$, Qiu-Ming Yao ${ }^{4}$, Babu Valliyodan ${ }^{2}$, Ming-Yi Bai ${ }^{5,6}$, Ming-Zhe Zhao ${ }^{2,7}$, Henng Ye $^{2}$, Zhi-Yong Wang ${ }^{5}$ and Henry T. Nguyen ${ }^{2^{*}}$

\begin{abstract}
Background: Brassinosteroids (BRs) play a crucial role in plant vegetative growth and reproductive development. The transcription factors BZR1 and BES1/BZR2 are well characterized as downstream regulators of the BR signaling pathway in Arabidopsis and rice. Soybean contains four BZR1-like proteins (GmBZLs), and it was reported that GmBZL2 plays a conserved role in BR signaling regulation. However, the roles of other GmBZLs have not been thoroughly studied, and the targets of GmBZLs in soybean remain unclear.

Results: In this study, we first characterized GmBZL3 in soybean from gene expression patterns, conserved domains in coding sequences, and genomic replication times of four GmBZL orthologous. The results indicated that GmBZL3 might play conserved roles during soybean development. The overexpression of GmBZL3 ${ }^{\text {P219L }}$ in the Arabidopsis BR-insensitive mutant bri1-5 partially rescued the phenotypic defects including BR-insensitivity, which provides further evidence that GmBZL3 functions are conserved between soybean and the homologous Arabidopsis genes. In addition, the identification of the GmBZL3 target genes through ChIP-seq technology revealed that BR has broad roles in soybean and regulates multiple pathways, including other hormone signaling, disease-related, and immunity response pathways. Moreover, the BR-regulated GmBZL3 target genes were further identified, and the results demonstrate that GmBZL3 is a major transcription factor responsible for BR-regulated gene expression and soybean growth. A comparison of GmBZL3 and AtBZR1/BES1 targets demonstrated that GmBZL3 might play conserved as well as specific roles in the soybean BR signaling network. Finally, the identification of two natural soybean varieties of the GmBZL3 mutantion by SNP analysis could facilitate the understanding of gene function during soybean development in the future.
\end{abstract}

Conclusions: We illustrate here that GmBZL3 orchestrates a genome-wide transcriptional response that underlies BR-mediated soybean early vegetative growth, and our results support that BRs play crucial regulatory roles in soybean morphology and gene expression levels.

Keywords: Soybean, GmBZL3, Brassinosteroid, ChIP-seq, Hormone crosstalk, SNP

\section{Background}

Brassinosteriods (BRs) play important and essential roles in a wide range of plant growth and development processes including cell elongation, cell division, plant architecture, photomorphogenesis, root development, photosynthesis, and senescence $[1,2]$. BRs also play significant roles in controlling flowering time, male fertility,

\footnotetext{
* Correspondence: nguyenhenry@missouri.edu

${ }^{\dagger} \mathrm{Li}$ Song and Wei Chen contributed equally to this work.

2Division of Plant Sciences, University of Missouri, Columbia, MO 65211, USA

Full list of author information is available at the end of the article
}

fruit ripening, seed development, seed filling and seed dormancy [3, 4]. In addition, BRs are essential for plant responses to various abiotic and biotic stresses, such as salt, drought, heat, cold, oxidative and heavy metal stresses, pathogen attacks and herbicide/pesticide tolerance $[5,6]$. The mechanisms of BR biosynthesis, signaling and response at the molecular level have been well characterized through genetics, proteomics and genomics technologies in Arabidopsis. The expression of BR biosynthetic genes is regulated by feedback from BR signaling to maintain balanced cell expansion in normal

(C) The Author(s). 2019 Open Access This article is distributed under the terms of the Creative Commons Attribution 4.0 International License (http://creativecommons.org/licenses/by/4.0/), which permits unrestricted use, distribution, and 
plant development [7-9]. The transcription factors, BZR1 and BZR2/BES1 have been reported to directly bind and regulate the expression of downstream target genes in the BR synthesis feed-back loop [10-12]. When the BR level is low, BZR1 is phosphorylated by the upstream BR signaling regulator BIN2 and then retained in the cytoplasm by the 14-3-3 proteins or degraded by the proteasome, which allows the expression of BR biosynthetic genes and then increases the level of BR $[13,14]$. When the BR level is high, dephosphorylated BZR1 translocates to the nucleus where it can bind to the target DNA regions and inhibits the expression of downstream BR-biosynthetic and BR-responsive genes [11, 15]. Several studies have linked BR signaling with numerous cellular processes by connecting the BR response genes and BZR1/BZR2 target genes $[15,16]$. In conclusion, BZR1 and BZR2/BES1 are master transcription factors that mediate $B R$ responsive gene expression, coordinating BR signaling, and BR biosynthesis and growth responses.

Soybean is a high protein and high oil crop that provides many products for humans and animals, including food. Several findings indicate that BRs play important roles during soybean growth and development. BRs dominate nodule formation in soybean roots and promote soybean epicotyl elongation [17-19]. The total nodulation, plant fresh weight, root length, shoot length, first internode length and lateral root numbers of soybean decreased in the 3 weeks after incubation with $0.1 \mu \mathrm{M}$ to $10 \mu \mathrm{M}$ of epibrassinolide [20]. Foliar application of BRs prior to drought stress could partially alleviate the detrimental effects of stress on the growth of soybean [21]. GmCPDs, genes that catalyze BR synthesis, are involved in the early stages of flowering regulation [22]. The pod size and plant height of the Arabidopsis mutant bri1-5 can be complemented by overexpressing GmBRI1, which suggests that GmBRI1 could function as a $B R$ receptor to mediate $B R$ signaling $[23,24]$. However, the gene regulatory network that links BR signals to various morphological and physiological responses remains largely unknown in soybean.

Recently, the function of GmBZL2 (AtBZR1-like gene) was studied in soybean. Overexpressed GmBZL2 $2^{\text {p216L }}$ Arabidopsis transgenic plants could partially rescue the defects of bri1-5 mutant and increase the seed number per silique, which reveals the involvement of GmBZL2 in a conserved BR signaling regulation pathway in Glycine max [25]. It was reported that soybean varieties with larger pods usually have higher GmBZR1 (GmBZL2) expression levels in pods [26]. These results suggest that understanding the roles of GmBZL2 in soybean BR signaling pathways can offer valuable information for enhancing soybean productivity. However, soybean contains four GmBZL genes, and the functions of other GmBZL genes remain unclear. Moreover, genome-wide studies have revealed that BZR1 and BES1/BZR2 directly regulate thousands of target genes in Arabidopsis, revealing a regulatory network of plant growth regulation $[15,16]$. A similar genome-wide study of GmBZL targets is required to elucidate the functions of BR in soybean.

In this study, GmBZL3 was first characterized in the soybean genome. The functions of GmBZL3 in BR signaling were investigated by overexpressing GmBZL3 and GmBZL $3^{\mathrm{P} 219 \mathrm{~L}}$ in the Arabidopsis bri1-5 mutant. Furthermore, our ChIP-seq analysis validated that GmBZL3 not only functions as a transcriptional regulator to mediate BR signal transduction but also functions as a hub to mediate BR crosstalk with other pathways. Finally, the natural variations in the GmBZL3 coding sequence were explored using soybean whole genome resequencing data, providing a good resource for gene functional analysis in the future.

\section{Results}

Characterization of GmBZL3 in the soybean genome

The four GmBZLs in G. max are divided into two subgroups internally based on phylogenetic tree analysis, which indicates that they may be from a specific duplication event during soybean evolution [25]. The soybean genome experienced segmental and tandem duplication events during evolution, causing gene family expansion, and the segmental duplication events in soybean occurred 59 and 13 million years ago (mya) [27]. Ka/Ks is the ratio between the number of nonsynonymous substitutions per nonsynonymous site $(\mathrm{Ka})$ and the number of synonymous substitution per synonymous site (Ks). Here, the $\mathrm{Ka} / \mathrm{Ks}$ ratios were calculated to evaluate the approximate duplication date of GmBZLs. We found that genome duplications of GmBZL1/2 and GmBZL3/4 clusters appear to have occurred at approximately 11 and 16 mya, respectively. Therefore, the duplication of GmBZL1/2 and GmBZL3/4 probably evolved through segmental duplication (Additional file 1). However, the duplicated GmBZL genes are under strong negative selection, as their $\mathrm{Ka} / \mathrm{Ks}$ ratios were estimated to be $<1$ (varied from 0.05 to 0.15 ).

Then, we analyzed the expression profiles of the GmBZL genes in different vegetative and reproductive tissues from published RNA-seq data sets [28]. As shown in the Additional file $2 \mathrm{~A}, G m B Z L 3$ transcripts accumulated at a high level in the pod, while GmBZL2 was highly expressed in the flower and GmBZL1/4 were highly expressed in the shoot apical meristem. Several reports have confirmed that AtBZR1 is engaged in various stress responses [5, 6, 29]. To gain more insight into the roles of GmBZLs under abiotic stress, gene expression patterns under various abiotic stress conditions (sodium, cold, dehydration and ABA) were further characterized using qRT-PCR (Additional file 2B-C). The 
results show that GmBZL3 expression levels are increased under both salinity and dehydration stresses, suggesting a role under abiotic stresses through transcriptional regulation.

To identify regions of similarity that may be a consequence of functional relationships, multiple amino acid sequence alignments were completed between AtBZR1/BZR2 and GmBZLs. As shown in Fig. 1, GmBZL3 contains highly conserved sequences including N-terminal DNA-binding domains, putative
14-3-3 binding sites, and sixteen putative BIN2 phosphorylation sites. Furthermore, GmBZL3 also contains the PEST domain (region rich in proline, glutamate, serine, and threonine), which share high similarity with that of the Arabidopsis genes. The conserved amino acid proline (Fig. 1, highlighted with the red color and boxes), which affects protein localization and phosphorylation status, was found in the PEST region of GmBZL3. All of the above observations suggest that

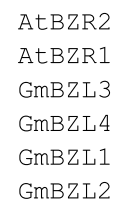

MTSDGATSTSAAAA-AAAMATRRKPSWRERENNRRRERRRRAVAAKIYTGLRAQGNYNLP MTSDGATSTSAAAAAAAAAAARRKPSWRERENNRRRERRRRAVAAKIYTGLRAQGDYNLP MTSDGA-------- TSAATHRRKPSWRERENNRRRERRRRAIAAKIYSGLRAQGNYNLP MTSDGA--------TSAATNRRKPSWRERENNRRRERRRRAISAKIYSGLRAQGNYNLP MADDGA--------TSAATSRRKPSWRERENNRRRERRRRAIAAKIYSGLRAQGNFNLP MVDDGA--------TSAATSRRKPSWRERENNRRRERRRRAIAAKIYSGLRAQGNFNLP

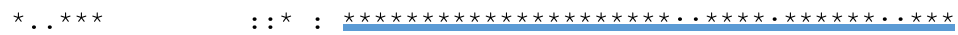

KHCDNNEVLKALCSEAGWVVEEDGTTYRKGHK-PLPGDMAGSSSRATPYS-SHNQSPLSS KHCDNNEVLKALCVEAGWVVEEDGTTYRKGCK-PLPGEIAGTSSRVTPYS-SQNQSPLSS KHCDNNEVLKALCAEAGWTVEEDGTTYRKGCRAPLPGDGVGTSTRNTPFS-SQNPSPLSS KHCDNNEVLKALCAEAGWAVEEDGTTYRKGCRAPYPGDGVGTSTRNTPFS-SQNPSPLSS KHCDNNEVLKALCAEAGWCVEEDGTTYRKGCKPPLA-NGAGSSMRNI PFSSSQNPSPLSS KHCDNNEVLKALCAEAGWCVEEDGTTYRKGCKPPLA-NGAGSSMRNITESSSQNPSPLSS

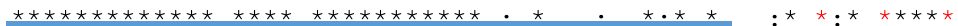

TFDSPILSYQVSPSSSSFPSPSRVGDPHNI-STIFPFLRNGGIPSSLPPIRISNSAPVTP AFQSPIPSYQVSPSSSSFPSPSRGE PNNNMSSTFFPFLRNGGIPSSLPSIRISNSCPVTP SFPSPIPSYQVSPSSSSFPSPSRLDA--NNPSNLIPYIRHA-FPSSLPPIRISNSAPVTP SFPSPIPSYQVSPSSSSFPSPSRLDA--NNPSNLIPYIRHA-FPASVPPIRISNSAPVTP SFPSPIPSYQVSPSSSSLPSPFRLDGDKDNVSNLIPYIRNA-S-LSLPPIRI SNSAPVTP SFPSPIPSYQVSPSSSSFPSPFRLDVDKDNVSHLIPYIRNA-S-LSLPPIRISNSAPVTP

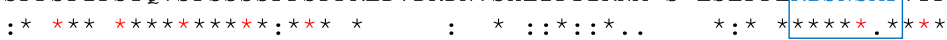

PVSSPTSRNPKPLPTWESFTKQSMSMAAKQSMTSLNYPFYAVSAPAS PTHHRQFHA PATI PVSSPTSKNPKPLPNWESIAKQSMAI-AKQSMASFNYPFYAVSAPASPTHRHQFHTPATI PLSSPTSRNPKPI PTWDSIAKASMAS----SFNHSHHPFFAASAPASPTHRHLY-APPTI PLSSPTSRNPKPI PTWDSIAKASMAS----SFNHSHHPFFAASAPAS PTHRHLY-APPTI PLSSPTSRNSKPIPTWES IAKESMAS--------FNYPFFAASAPASPTHRHLY-TPLTI PLSSPTSRNPKPIPTWESIAKESMAS--------FSYPFFAASAPASPTHRHLY-TPPTI

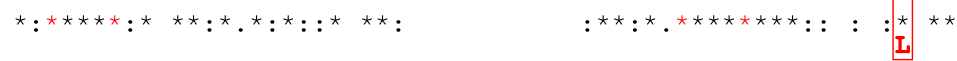

PECDESDSSTVDSGHWISF QKFAQQQPFSASMVPTSPTFNLVKPAPQQLSPNTAA----PECDESDSSTVDSGHWISFQKFAQQQPFSASMVPTSPTENLVKPAPQQMS PNTAA----PECDESDTSTVESGQWLNFQAFAP----SVSAVPISPTMNFIKPVVSQQHKHNLNLSGNG PECDESDTSTVESGQWLNFQAFAP----SVSPVPISPTMNFIKPVVSQQHKHNLNLPGNG PECDESDTSIGESGQWVKFQAFAP----SASVEPTSPTENLVKPVIPHR------MPDNS PECDESDTSTGESGQWVKFQAFAP----SSSVLPISPTFNLVKPVVPPG-------MPDNS

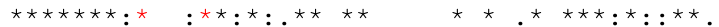

IQEIGQSSEFKFENSOVKPWEGERIHDVAMEDLELTLGNGKAHS * FQEIGQSSEFKFENSQVKPWEGERI HDVGMEDLELTLGNGKARG* IQEMRISE--PEFAMQVKPWVGERIHEVGLDDLELTLGSGKTPA* IQEMRISE--PEFAMQVKPWVGERIHEVGLDDLELTLGSGKTPA* IQVMRTSS--EEFGVQVKPWVGEKIHEVALDDLELTLGSGKVRS* IQEMRTSS--DEFGVQVKPWVGEKIHEVALDDLELTLGSGKVRS *

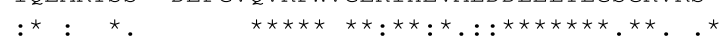

Fig. 1 Multiple amino acid sequence alignment of the GmBZL in Soybean and Arabidopsis. The marked features are the N-terminal DNA binding domain (blue underline), putative 14-3-3 binding site (blue letters and box), putative sites of phosphorylation by GSK-3 kinase (red star), and the PEST domain (green letter and over line). The conserved amino acid (proline) between Arabidopsis and soybean in the PEST region is indicated by a red box, and was mutated to the amino acid leucine for the validation of conserved functional 
GmBZL3 plays a conserved role in the BR signaling pathway in soybean.

\section{Cross-species complementation test of GmBZL3 in Arabidopsis}

A dominant bzr1-1D mutation increases BZR1 protein accumulation, suppresses BR-insensitive mutant (bri1) phenotypes, and enhances the feedback inhibition of BR biosynthesis in Arabidopsis [10]. The nuclear localization and phosphorylation ratio of GmBZL2 induced by BR and overexpressed GmBZL2 ${ }^{\mathrm{P} 216 \mathrm{~L}}$ (containing the conserved proline site mutation) in Arabidopsis Col-0 could produce the bzr1-1D phenotypes [25]. Cross-species complementation experiments were performed here to test whether the GmBZL3 gene is a functionally conserved regulator in the BR signaling pathway. Transgenic Arabidopsis plants were generated to overexpress
GmBZL3 or GmBZL3 ${ }^{P 219 L}$ (containing a proline-to-leucine mutation) in the bri1-5 mutant. As indicated in Fig. 2, no distinct difference in morphological phenotype was observed between the GmBZL3/bri1-5 transgenic plants and the bri1-5 mutant (Fig. 2a). Furthermore, the GmBZL3/bri1-5 plants showed a similar phenotype as the bri1-5 mutant under either dark or light conditions during the seedling stage (Fig. 2b-e). However, the GmBZL3 $3^{P 219 L} /$ bri1-5 transgenic plants showed a bzr1$1 D$-like phenotype with improved plant height at the mature stage under normal conditions (Fig. 2a). The

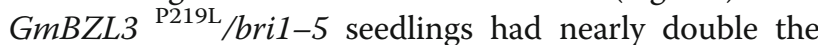
hypocotyl length under dark conditions compared with that of bri1-5 mutant seedlings (Fig. $2 \mathrm{~b}$ and c). Under light conditions, the root growth of GmBZL3 ${ }^{\text {P219L }} /$ bri1-5 seedlings was longer than that of the bri1-5 mutant (Fig. 2d and e). Interestingly, even higher RNA expression levels of GmBZL3 in GmBZL3/bri1-5 compared
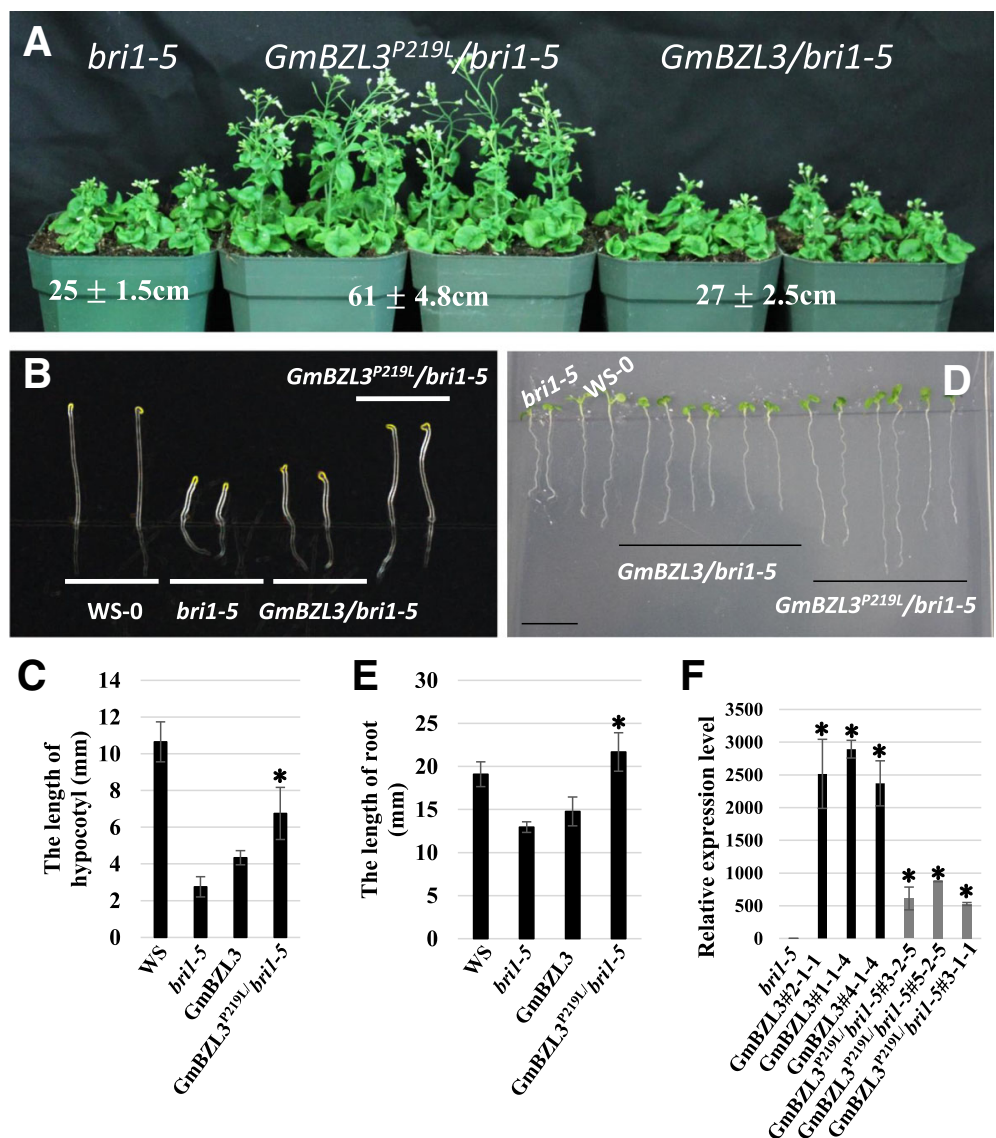

Fig. $2 \mathrm{GmBZL}^{\mathrm{P} 219 \mathrm{~L}}$ rescues the bri1-5 insensitive phenotype in transgenic plants. GmBZL3/bri1-5 indicates overexpressed GmBZL3 in the bri1-5 mutant. GmBZL3\#2-1-1, GmBZL3\#1-1-4 and GmBZL3\#4-1-4 are the three independent lines in which GmBZL3 is overexpressed in the bri1-5

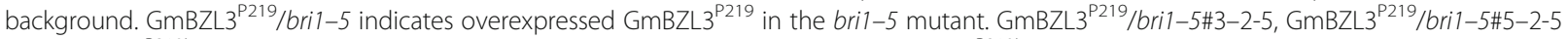
and GmBZL3 ${ }^{\text {2219L } / b r i 1-5 \# 3-1-1 ~ a r e ~ t h e ~ t h r e e ~ i n d e p e n d e n t ~ l i n e s ~ t h a t ~ o v e r e x p r e s s ~ G m B Z L 3 ~}{ }^{\text {P219L }}$ in a bri1-5 background. a The plant height of a one-month-old bri1-5 mutant was significantly rescued by overexpressing GmBZL $3^{\text {P219 }}$. b-c Comparison of the length of the hypocotyl of 4-day seedlings (grown in the dark) in various plant lines. d-e Comparison of the length of the primary root of 7-day seedlings (grown under light) in various plant lines. $f$ Transgene expression in various lines revealed by quantitative RT-PCR. Significant difference between the compared values are shown $(P<0.05)$. Error bars indicate S.D. $(n \geq 30)$ 
with GmBZL3 ${ }^{\mathrm{P} 219 \mathrm{~L}} /$ bri1-5 were found (Fig. 2f). Taken together, these results demonstrate that GmBZL3 can regulate BR signaling when heterologously expressed in Arabidopsis and thus is functionally conserved.

Previous studies showed that AtBZR1 and GmBZL2 could partially rescue the BR-sensitivity phenotypes of bri1-5 mutant in Arabidopsis [10, 25]. As shown in Fig. 3a, the root length of wild type was significantly inhibited by exogenous $100 \mathrm{nM} \mathrm{BL}$. However, the root length of bri1-5 was increased under lower concentrations of BL (1-100 nM). We also found that overexpressed GmBZL $3^{P 219 L}$ partially rescued the bri1-5 insensitivity phenotype at both $10 \mathrm{nM}$ and $100 \mathrm{nM}$ (Fig. 3b). The insensitivity phenotype was significantly rescued in the GmBZL $3^{P 219 L} / \mathrm{bri1}-5$ seedlings compared with bri1-5 under $100 \mathrm{nM}$ BL treatments. This result illustrates that the conserved amino acid mutation (P219L) in the PEST motif of GmBZL3 led to the altered BR response phenotypes.

\section{ChIP-seq results reveal that GmBZL3 regulates a broad} range of biological processes and cellular activities

A ChIP-seq experiment was carried out to identify genes directly regulated by native GmBZL3 in soybean using an anti-GmBZL3 antibody. Since the pre-immunization serum was not used as a negative control, ChIP-qPCR analysis was conducted by using the immunoprecipitation of DNA before resequencing. Four genes (DWF4, BR6ox2, CPD, BAS1) that may act as GmBZL3 targets were selected. PROTEIN PHOSPHATASE 2A (PP2A) mediates the dephosphorylating and activation of BZR1 by protein interaction was used as negative control. As shown in Additional file 3, the amount of DWF4 fragment significantly enriched in the immunoprecipitation of DNA compared with that of PP2A. No significant difference was found in other three putative targets.

Furthermore, 5185 binding peaks were identified in two independent biological repeats. These binding sites were linked to 2923 nearest neighbor genes, which were located on all 20-soybean chromosomes. Among these, 729 genes with high peaking scores were considered high-confidence GmBZL3-binding target genes (Additional file 4) and included in the subsequent studies. GO term analysis indicated that 407 genes were involved in different biological processes (Fig. 4), and 322 genes were not assigned to any known process. Among the enriched biological processes, RNA (regulation of transcription) and protein composed the largest subgroups. Genes related to transport (26), development (22) signaling (39), miscellaneous (33) and

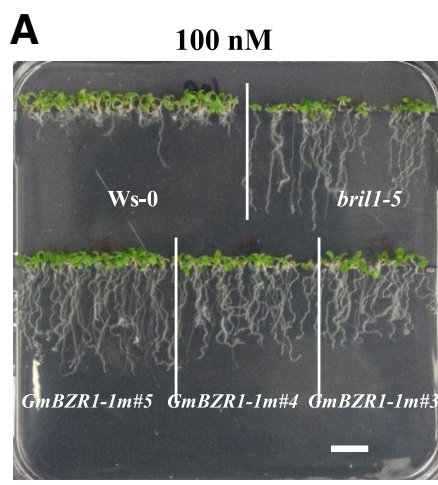

B

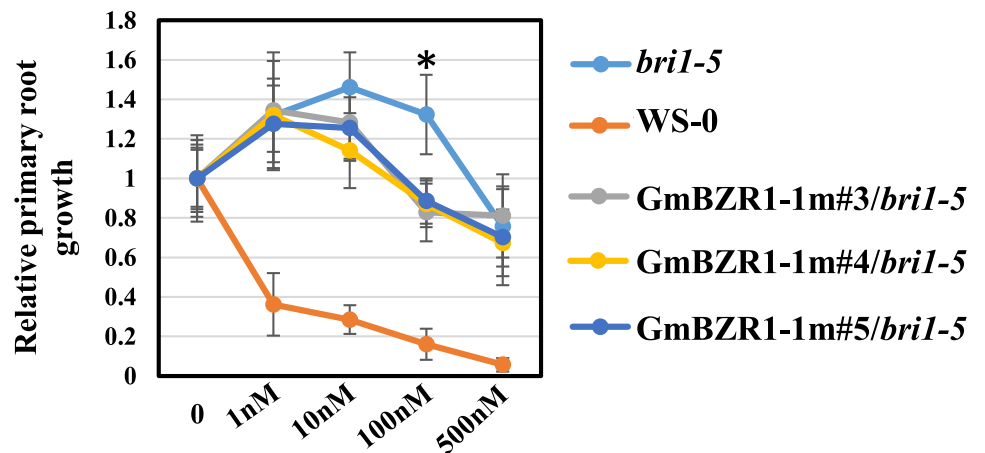

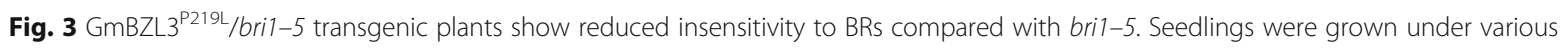
concentration of epibrassinolide for 7 days in $16 \mathrm{~h}$ light/ $8 \mathrm{~h}$ dark condition. The root length was measured. Significant difference between the compared values are shown $(P<0.05)$. Error bars indicate S.D. $(n \geq 30)$ 


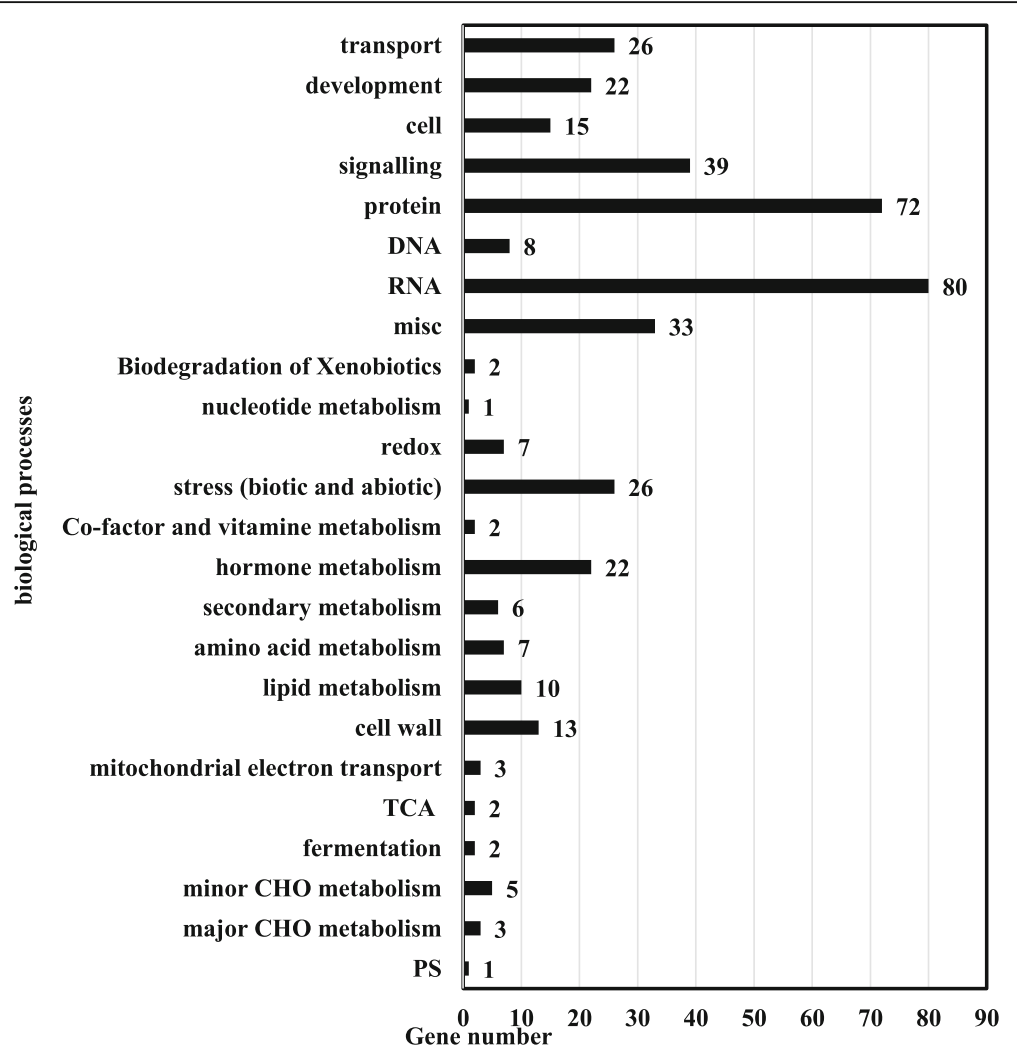

Fig. 4 Functional categorization of GmBZL3-targeted genes based on biological processes. The identified high-ranking GmBZL3 targets are grouped based on MapMan "Bin" and GO ontology. RNA and protein processes related targets were significantly enriched. Gene numbers are displayed next to the terms. The abscissa indicates the number of targets in each category and the ordinate indicates the enriched $\mathrm{GO}$ categories in biological processes

stress (26) represented the major categories, indicating that GmBZL3 plays multiple roles during soybean development. A small number of genes in the categories of secondary metabolism (6), amino acid metabolism (7), lipid metabolism (10), and carbohydrate metabolism (8) were also found. According to the molecular functional distribution, a total of seven categories were found to be highly and significantly enriched in genes involved in DNA binding, sequence-specific DNA binding transcription factor activity, protein binding, transport activity, etc., (Additional file 5, $p<0.05$ ). These results indicate that GmBZL3 is a major transcription factor responsible for gene expression and soybean growth.

\section{GmBZL3 directly regulates genes that function in the synthesis and response of other hormones}

Crosstalk between BR and other hormones has been observed at both the physiological level and the gene expression level in Arabidopsis [30-34]. However, functional interactions among hormones remain largely unknown in soybean. In this study, we found some genes related to auxin signaling, response and synthesis that were directly targeted by GmBZL3, including SAUR-like protein, GH3 and YUCCA3 (Table 1). Directly target genes were also been identified for ethylene metabolism and regulation of ethylene responsive pathways (Glyma.06G221800 and Glyma.18G164100). In addition, GmBZL3 targeted a number of gibberellin synthesisdegradation-related genes and GA-regulated family genes (Glyma.13G039600 and Glyma.13G069900). Several Jasmonate (JA) pathway-related genes, including $L O X 2$, AOC4 and OPR2, were identified in our ChIP-seq experiments. JAZ1 is a coreceptor and repressor of JA signaling in Arabidopsis [35]. Here, we found that GmBZL3 could bind the promoter region of two JAZ1 genes, which may indicate a negative relationship between BR and JA. These results demonstrated that the crosstalk between $\mathrm{BR}$ and other hormones existed in soybean and that GmBZL3 acts as an intermediate factor to mediate hormonal crosstalk.

\section{GmBZL3 mediates crosstalk between BR and other development pathways}

In Arabidopsis, the hormonal interactions between BR and abscisic acid, ethylene or salicylic acid participate in 
Table 1 Representative target genes with high-ranking score ( $>70)$ that potentially regulated by GmBZL3 transcription factor in soybean were identified through ChIP-seq method

\begin{tabular}{|c|c|}
\hline Annotation & Identifier \\
\hline Expansin & $\begin{array}{l}\text { Glyma.06G246400 Glyma.02G109100 } \\
\text { Glyma.06G195000 Glyma.14G203900 }\end{array}$ \\
\hline Auxin efflux carrier family protein & Glyma.13G038300 \\
\hline Auxin-responsive $\mathrm{GH} 3$ family protein & Glyma.02G125600 \\
\hline IAA-leucine-resistant (ILR1)-like 3 & Glyma.04G226600 \\
\hline SAUR-like auxin-responsive protein family & Glyma.11G096800 Glyma.18G110300 \\
\hline YUCCA 3 & Glyma.20G080000 \\
\hline Dormancy/auxin associated family protein & Glyma.20G237200 \\
\hline Jasmonate-zim-domain protein 1 & Glyma.09G071600 Glyma.15G179600 \\
\hline Gibberellin 20-oxidase 3 & Glyma.14G157400 \\
\hline Gibberellin 2-oxidase 4 & Glyma.07G236100 Glyma.17G037300 \\
\hline Gibberellin 2-oxidase 8 & Glyma.05G081600 \\
\hline Gibberellin-regulated family protein & Glyma.13G039600 Glyma.13G069900 \\
\hline BAK1-interacting receptor-like kinase 1 & Glyma.03G095700 Glyma.08G062800 \\
\hline Brassinosteroid signalling positive regulator (BZR1) family protein & Glyma.06G034000 \\
\hline Disease resistance protein (CC-NBS-LRR class) family & $\begin{array}{l}\text { Glyma.05G082200 Glyma.05G082500 Glyma.01G183400 } \\
\text { Glyma.17G180300 }\end{array}$ \\
\hline Disease resistance protein (TIR-NBS-LRR class) family & Glyma.13G194900 \\
\hline Disease resistance-responsive (dirigent-like protein) family protein & $\begin{array}{l}\text { Glyma.03G147900 } \\
\text { Glyma.19G151100 }\end{array}$ \\
\hline LRR and NB-ARC domains-containing disease resistance protein & Glyma.03G039300 \\
\hline NB-ARC domain-containing disease resistance protein & Glyma.01G065800 \\
\hline SCARECROW-like 13 & Glyma.07G266500 \\
\hline Senescence associated gene 20 & Glyma.11G163100 \\
\hline Stachyose synthase & Glyma.19G217700 \\
\hline Fatty acid biosynthesis 1 & Glyma.15G181500 \\
\hline Flowering promoting factor 1 & Glyma.17G202100 \\
\hline Nitrate transporter 1.1 & $\begin{array}{l}\text { Glyma.05G056900 Glyma.01G042100 } \\
\text { Glyma.02G022200 }\end{array}$ \\
\hline WRKY DNA-binding protein & $\begin{array}{l}\text { Glyma.05G127600 Glyma.06G219800 } \\
\text { Glyma.02G232600 Glyma.14G200200 }\end{array}$ \\
\hline WRKY family transcription factor & Glyma.13G117600 Glyma.19G254800 \\
\hline Dof-type zinc finger DNA-binding family protein & $\begin{array}{l}\text { Glyma.15G071400 Glyma.15G082400 } \\
\text { Glyma.19G199200 }\end{array}$ \\
\hline $\begin{array}{l}\text { NAC (No Apical Meristem) domain transcriptional regulator superfamily } \\
\text { protein }\end{array}$ & $\begin{array}{l}\text { Glyma.05G195000 Glyma.06G248900 } \\
\text { Glyma.12G149100 Glyma.12G226500 }\end{array}$ \\
\hline NAC transcription factor-like 9 & Glyma.06G138100 \\
\hline PIF1 helicase & $\begin{array}{l}\text { Glyma.10G079800 Glyma.13G024400 } \\
\text { Glyma.14G128000 }\end{array}$ \\
\hline DnaJ/Hsp40 cysteine-rich domain superfamily protein & Glyma.14G104200 \\
\hline Heat shock cognate protein $70-1$ & Glyma.03G171100 \\
\hline Heat shock protein DnaJ with tetratricopeptide repeat & Glyma.19G215400 \\
\hline Heat shock transcription factor B2A & Glyma.16G196200 \\
\hline
\end{tabular}

stress-related development [29]. However, the molecular mechanism of the involment of BR in stress tolerance remains poorly understood in soybean. It has been reported that BZR1 mediates the antagonism between immunity signaling and BRs by inducing the expression of several WRKY transcription factors that negatively 
control early immune responses [36]. Here, we found that several disease resistances-related genes and WRKY transcription factors were targeted by GmBZL3. BR controls plant growth by acting on both expansion and division in the leaf [37]. The AtBZR1 protein accumulates in the growing region of Arabidopsis hypocotyl under dark conditions, indicating that BZR1 plays a role in cell expansion [10]. Studies of the Arabidopsis bzr1-1D mutant suggested that BZR1 is involved in growth promotion [10]. Here, we found that GmBZL3 directly regulated the expression of four expansin genes (Table 1). Several targets involved in drought and heat stress responses, such as heat shock protein, heat shock transcription factor, and ERD15 (early responsive to dehydration 15), were found. Moreover, senescence, fatty acid biosynthesis and stachyose synthase related genes were regulated by GmBZL3. These results not only confirmed that a similar BR regulatory mechanism existed in Arabidopsis and soybean but also suggested that the transcription factor GmBZL3 directly linked BR signal transduction with soybean development.

\section{The expressions of GmBZL3 targets were tightly regulated under different $B R$ levels in soybean}

Propiconazole (Pcz), a BR biosynthesis inhibitor, inhibits BR metabolism and induces BR deficiencies in Arabidopsis, maize and soybean seedlings [38], (Song et al., unpublished). The expression levels of BR response genes are precisely regulated by BR concentration $[11,12,16]$. Genome-wide gene expression analysis was conducted by using stage V1 (first-node: fully developed leaves at unifoliolate node) soybean seedlings exposed to higher concentrations of BR synthesis inhibitor with/without epi-brassinolide. Briefly, V1 stage soybean plants were irrigated with water containing high concentration BR synthesis inhibitor $(5 \mu \mathrm{M}$ propiconazole for 10 days, Banner Maxx-60,207-90-1, Syngenta, Greensboro, NC), or a combined treatment of high concentration inhibitor and low concentration brassinolide (BL) $(5 \mu \mathrm{M}$ Pcz with $10 \mathrm{nM}$ BL for 10 days, Pcz-BL). High concentration BL was applied for $1 \mathrm{~h}$ or $8 \mathrm{~h}$ after 10 days treatment with high concentration inhibitor $5 \mu \mathrm{M}$ Pcz for 10 days then with $1 \mu \mathrm{M}$ BL $1 \mathrm{~h}$, Pcz-BL-1 h; $5 \mu \mathrm{M}$ Pcz for 10 days then with $1 \mu \mathrm{M}$ BL for $8 \mathrm{~h}$, Pcz-BL-8 $\mathrm{h}$ ) (Song et al., unpublished). To characterize the genome-scale adjustment of GmBZL3 targets during soybean development, the overlap between GmBZL3 targets and BR-regulated genes was examined.

We found that the transcript abundance of 154 targets was significantly altered under at least one treatment condition (Fig. 5a). In addition, the transcript levels of some GmBZL3 targets were induced, while others were repressed under the same concentration of BR. Moreover, a number of genes were significantly differentially regulated under a specific treatment, but there was no obvious change in other treatments. Six target genes of GmBZL3 were further selected to verify the expression pattern at different BR levels using qRT-PCR. As shown in Fig. 5b, a stachyose synthase gene (Glyma.19G21770) and a heat shock protein (Glyma.03G171100) were significantly reduced by $5 \mu \mathrm{M}$ Pcz followed by $1 \mu \mathrm{M}$ BL for $8 \mathrm{~h}$. An expansin gene (Glyma.01G050100) was reduced under $5 \mu \mathrm{M}$ Pcz with or without I nM BL combination treatment. However, the expression of this expansin gene could be recovered by applying $1 \mu \mathrm{M}$ BL for 1 or $8 \mathrm{~h}$. These results indicated that the expression patterns of GmBZL3 targets were specifically controlled by different BR levels in soybean and that GmBZL3 plays an important regulatory function during the soybean response to different BR levels.

\section{Comparison of GmBZL3 and AtBZR1/BES1 targets}

ChIP-chip studies have determined that BZR1 and BZR2/BES1 directly regulate over thousands of genes, revealing the brassinosteroid transcription network in Arabidopsis $[15,16]$. To reveal the evolutionary variation of the BR gene network between Arabidopsis and soybean, we first compared 3410 BZR1 high-confidence targets with 729 GmBZL3 high-confidence target homologs. Our results showed that 162 out of 729 (22.2\%) GmBZL3 targets were identical to BZR1 targets (Fig. 6a). Furthermore, 140 out of 729 (19.2\%) GmBZL3 target genes were overlapped with BZR1 low-confidence targets. Therefore, a total of $302(41.4 \%)$ GmBZL3 high-confidence target homologs were matched with BZR1 targets. However, only 53 (7.27\%) overlapping genes were found between GmBZL3 target homologs and BES1 targets (Fig. 6b). Together, these analyses demonstrated that GmBZL3 might play conserved as well as specific roles in the soybean BR signaling network similar to the role of BZR1 in Arabidopsis. Furthermore, the small number of overlapping targets between GmBZL3 and BES1 may imply that there are different functions between soybean and Arabidopsis.

The Analyses of these overlapping genes between the GmBZL3 target homologs and the BZR1 targets showed a wide range of cellular transport, cell wall-related enzymes, transcription factors, metabolism, stress, and hormone-related genes (Fig. 6c). For example, EXPA1 and EXPA8, which likely mediate the BR responses of cell elongation and differentiation, were targeted by both GmBZL3 and BZR1. The gibberellin synthesis-degradation-related genes, GA20-oxidase and GA2-oxidase were regulated by both GmBZL3 and BZR1. These results indicated that several developmental processes are conserved in the BR response networks of soybean and Arabidopsis and revealed the nodes of crosstalk between $B R$ and other regulatory pathways. 


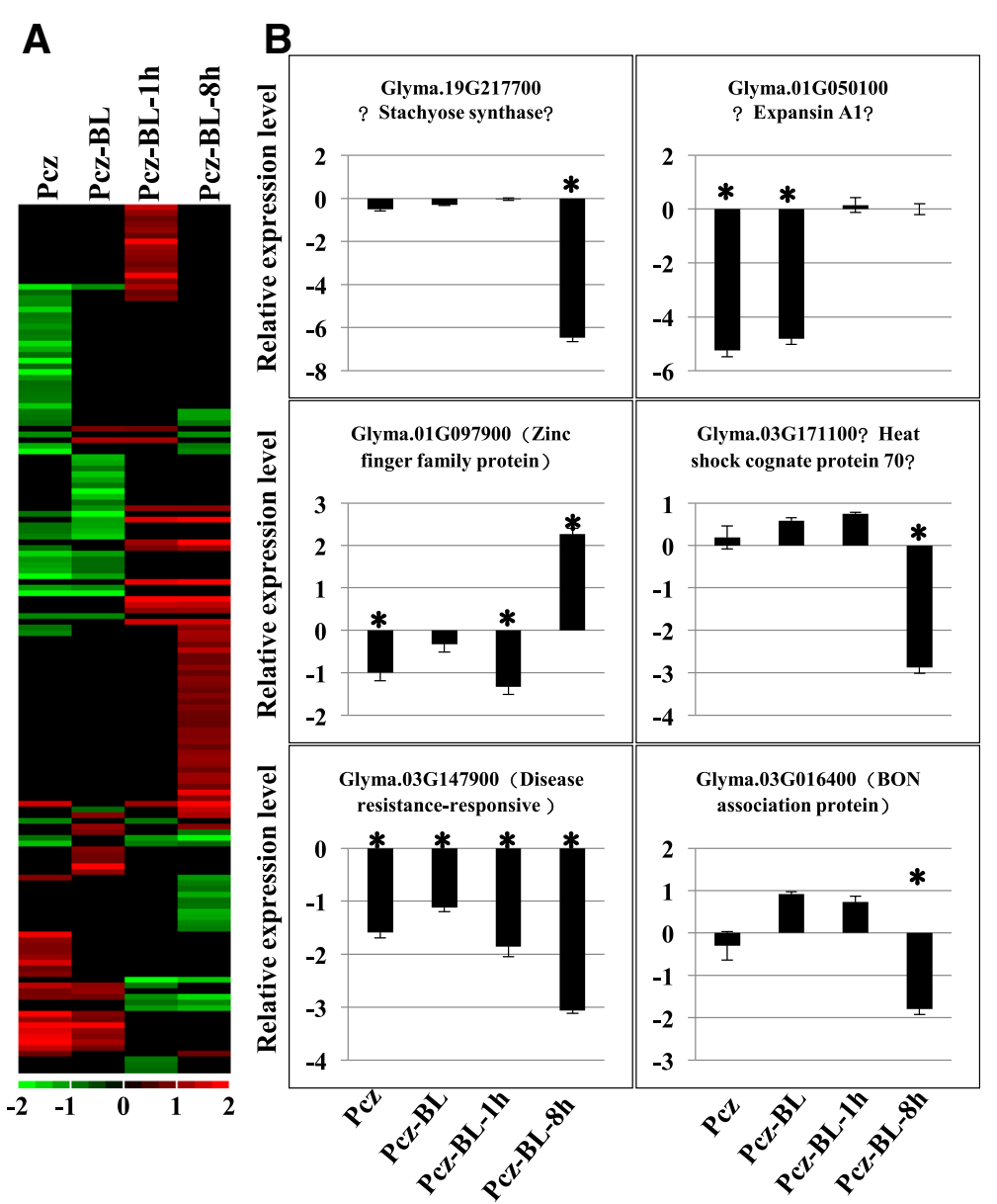

Fig. 5 Expression patterns of GmBZL3 target genes in response to a BR inhibitor in combination with or without epibrassinolide. a Heatmap representation of expression patterns of different GmBZL3 targets in soybean Williams 82 under following conditions (PCZ: $5 \mu$ M PCz for 10 days. PCz-BL: $5 \mu \mathrm{M}$ PCz with $10 \mathrm{nM}$ BL for 10 days. Pcz-BL-1 h: $5 \mu \mathrm{M}$ PCz for 10 days then with $1 \mu \mathrm{M}$ BL for $1 \mathrm{~h}$. Pcz-BL-8 h: $5 \mu \mathrm{M}$ PCz for 10 days then with $1 \mu \mathrm{M}$ BL for $8 \mathrm{~h}$ ). The expression data values were median-centered and normalized for each gene before transforming to the color scale (log2transformed ratios). The color bar at the bottom shows the range of expression values from highest expression level (red) to lowest expression level (green). 0 is the median expression level (Black). $\mathbf{b}$ qRT-PCR analysis of six GmBZL3 target genes was performed using total RNA isolated from Wm82 seedlings under control. Pcz. Pcz-BL. Pcz-BL-1 h and Pcz-BL-8 h treatments. Relative gene expression levels (fold change, log2) are shown following normalization with actin (Glyma.18G290800) transcript values. Error bars represent the standard error of the mean. The $y$-axis represents the relative gene expression level in different samples. Three independent experiments were performed. A representative result is shown. The star $\left(^{*}\right)$ indicates statistically significant differences among the means $(p<0.05)$

\section{Exploring natural variations in the GmBZL3 coding} sequence using soybean whole genome resequencing data To understand the genetic variations of GmBZL3 in different soybean varieties, single nucleotide polymorphisms (SNPs) were identified in 106 soybean genotypes by using whole genome resequencing data [39]. Only two synonymous SNPs were observed in the coding region of GmBZL3 compared with the reference genome sequence (Williams 82) (Additional file 6). However, one nucleic acid deletion was found in the soybean lines PI594599 and PI603154. This nucleic acid gap causes an amino acid frame shift and introduces a premature stop codon in these two soybean genotype lines. Specifically, the conserved PEST sequence in GmBZL3 can be completely changed due to this deletion. These identified soybean lines can be used as mutant sources for further functional characterization of the GmBZL3 gene in the soybean BR signaling pathway.

\section{Discussion}

We illustrate here that GmBZL3 orchestrates a genomewide transcriptional response that underlies BR-mediated soybean early vegetative growth, and our results support that BRs play crucial regulatory roles in many processes ranging from morphology to gene expression levels. A cross-species complementation test in Arabidopsis demonstrated that GmBZL3 plays conserved and broad roles in regulating BR signaling. 

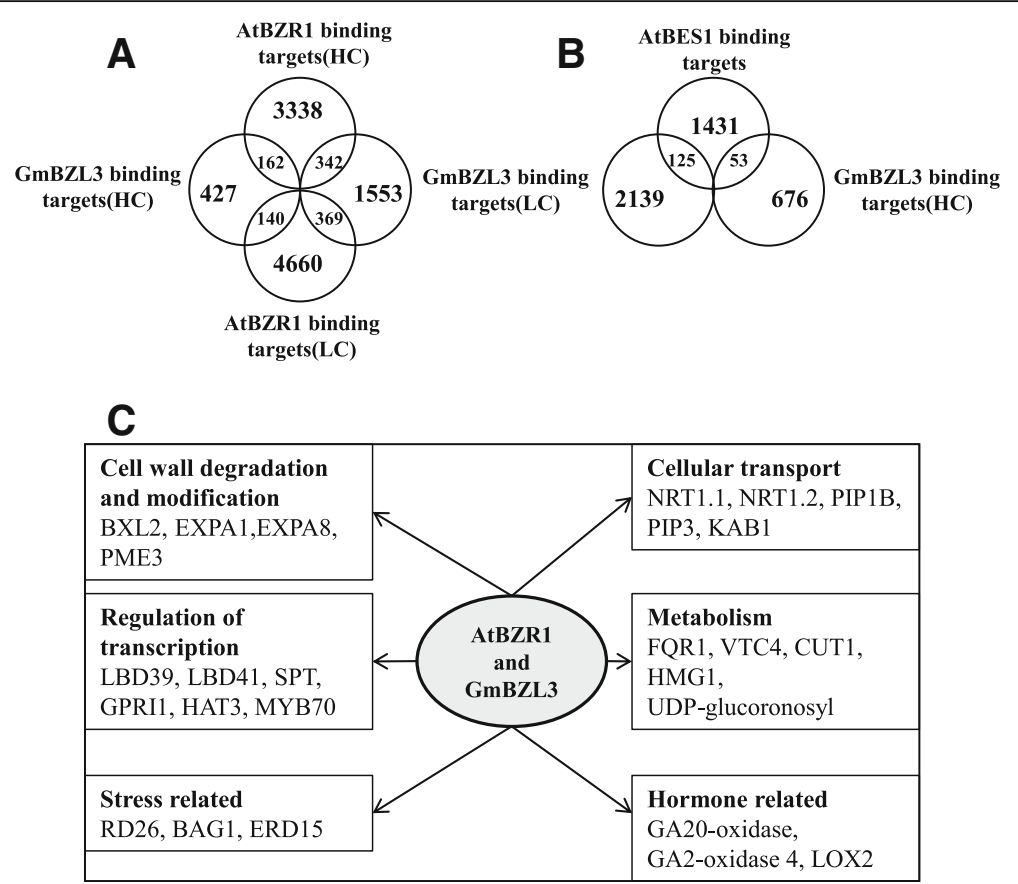

Fig. 6 Comparison of GmBZL3 and AtBZR1/BES1 targets. a-b Venn diagram showing the overlap of GmBZL3 target genes with BZR1 or BES1 targets. HC: high-confidence. LC: low-confidence. c Representative overlaps of GmBZL3 target genes with BZR1 targets in various cellular, response and metabolic pathways. NRT1.1 (Nitrate transporter 1.1). NRT1.2 (Nitrate transporter 1.2). PIP1B (Plasma membrane intrinsic protein 1;2). PIP3 (Plasma membrane intrinsic protein 3). KAB1 (Potassium channel beta subunit). FQR1 (Flavodoxin-like quinone reductase 1). VTC4 (Inositol monophosphatase family protein). CUT1 (Cuticular 1). HMG1 (3-hydroxy-3-methylglutaryl coa reductase). LOX2 (Lipoxygenase 2). BXL2 (Beta-xylosidase 2). EXPA1 (Expansin A1). EXPA8 (Expansin A8). PME3 (Pectin methylesterase 3). LBD39 (lateral organ boundaries domain protein 39). LBD41 (lateral organ boundaries domain protein 41). SPT (SPATULA). GPRI1 (GOLDEN2-like 1). HAT3 (Homeobox-leucine zipper protein 3). AtMYB70 (Myb domain protein 70). RD26 (Responsive to dessication 26). BAG1 (BCL-2-associated athanogene 1). ERD15 (Early responsive to dehydration 15)

\section{Gene sequence and structure characteristics of GmBZL3} AtBZR1 and AtBES1/BZR2 in Arabidopsis have a high similarity of $88 \%$ identity in overall amino acid sequence and $97 \%$ identity in the $\mathrm{N}$-terminal DNA binding domain [10]. In addition, AtBZR1 and AtBZR2/BES1 bind to similar DNA sequences, such as the E-box (CANNTG) and BRRE (CGTGT/CG) motifs [11, 12, 16]. These findings of high sequence similarity indicate that the BZR1 gene family may play conserved biological functions. In soybean, four GmBZR1 orthologues show similar sequence domains to $A t B Z R 1$ and $A t B E S 1 / B Z R 2$. It was reported that the PEST sequence serves as a proteolytic signal regionthat targets proteins for rapid degradation [40]. A PEST sequence located in the BZR protein sequence was identified in Arabidopsis, rice and soybean [10, 25, 41]. A proline-to-leucine mutation in the PEST domain leads to the accumulation of BZR1 and altered BR responsive phenotypes in bzr1-1D and bes1-D mutants [41]. The PEST sequence mutation in bzr1-1D increases its interaction with PP2A phosphatase and enhances dephosphorylation of the BZR1 protein [42], which reduces its binding affinity to the 14-3-3 protein as well as increases its nuclear accumulation and DNA binding [13]. The bzr1-1D allele reverses the dwarf phenotype when transformed into the bri1-5 mutant, whereas the overexpression of the wild type BZR1 in either WT or bri1-5 mutant plants did not cause obvious phenotypic changes due to the tight control of BZR1 activity by phosphorylation [10]. The effect of a conserved mutation located in the PEST motif of GmBZL3 was confirmed through functional validation in transgenic Arabidopsis plants (Fig. 3). The C-terminal domain is required for interactions between BZR2/BES1 and BIN2 or BIM1 in vivo [12, 43]. In this study, 16 putative BIN2 phosphorylation sites were identified in GmBZL3. Shortened hypocotyls, roots, and stems are the most notable morphological characteristics of the Arabidopsis BR receptor mutants. The overexpression of GmBZL3 ${ }^{\text {P219L }}$ could rescue the stem, hypocotyl, and root lengths, but could not rescue the BR insensitivity, as expected. These results demonstrate that GmBZL3 is a functional transcription factor involved in the BR signal transduction cascade.

\section{GmBZL3 targets response to BR signaling}

A large numbers of overlapping target genes regulated by AtBZR1 and AtBZR2/BES1 were identified by combining 
the chromatin-immunoprecipitation microarray (ChIP-chip) and RNA-seq/microarray results [15, 16]. Genomewide studies have further revealed that the BES1/BZR1 family regulates a transcription network involving thousands of genes. Approximately one-quarter of BR-responsive genes are directly regulated by BZR1/BZR2 (approximately 1000 genes) $[15,16]$. In our study, more than two thousand target genes bind by GmBZL3 were identified through ChIP-seq experiments, which indicated that GmBZL3 is a major regulator of the BR pathway. In addition, many GmBZL3 targets showed increased or decreased of expression levels in response to different BR levels based on four sets of RNA-seq data. Therefore, it is conceivable that GmBZL3 has a dual regulatory function under different BR levels.

\section{Comparison of GmBZL3 and BZR1 targets reveals interesting features of gene regulation}

It has been reported that BR is involved in drought stress in a range of plant species [44-46]. BR signaling transduction-related receptors and regulators play important roles under drought conditions [47-49]. Recently, it was found that a NAC transcription factor, RD26 (Responsive to desiccation 26), mediates crosstalk between drought-related and BR signaling pathways by coordinating the transcriptional activities of RD26 and BES1 [50]. Two soybean genes (Glyma.06G248900 and Glyma.12G149100) encoding RD26 were targeted by GmBZL3 in our results and were significantly induced under Pcz and Pcz-BL treatment (data not shown). Previous studies indicated that ERD15 is a negative regulator of the ABA-response gene in Arabidopsis [51]. Here, we also found that ERD15 was targeted by GmBZL3 and BZR1 in soybean and Arabidopsis, respectively. Moreover, GmBZL3 and BZR1 targeted two water channel proteins (PIP1B and PIP3) in soybean and Arabidopsis, respectively. Therefore, our data not only support the conclusion that GmBZL3 plays a role in soybean drought response, but also provide candidate genes for elucidating the molecular mechanism in the future.

The de-etiolation phenotype of BR mutants indicates that BR plays an essential role in photomorphogenesis and many of the genes regulated by BZR1 overlap with the targets of HY5 and PIL5 (light-signaling pathway key transcription factor) $[16,52]$. However, few light-related genes were found in GmBZL3 targets. This result demonstrates that GmBZL3 may play indirect and specific roles in the light signaling pathway.

\section{Soybean whole genome sequencing reveals good mutation resources for GmBZL3}

It is difficult to validate gene functions by transgenic methods since soybean transformation efficiency is low [53]. Therefore, soybean genes are often transformed into other plants, such as Arabidopsis or tobacco, for functional evaluation. To date, hairy root transformation has offered an effective alternative to obtain a high number of soybean transformants in a relatively short time for gene function validation. Although this system has made significant contributions to soybean root studies, it is questionable in the case of characterization of nonroot traits [54-56]. Luckily, there are several mutations in nature, such as a null soybean mutant or recessive alleles for target genes, which can be used as valuable genetic sources. The first complete reference genome for soybean, Williams 82, was released in 2010 [27]. Hyten et al. [57] developed an initial version of SNP detection assays to identify a large number of SNPs and constructed a genetic map with higher resolution. A large amount of genome sequencing data has been generated with recently published GWAS studies [39, 58, 59]. These genomic resources will provide a range of opportunities for soybean improvement through understanding gene function by map-based cloning and reverse genetic approaches.

In our study, two mutant lines of GmBZL3 were identified by analyzing whole genome resequence data. These lines will be excellent materials for further gene functional characterization. Moreover, the marker-assisted backcrossing strategy can be used to develop GmBZL3 mutants suitable for gene function validation. It has been reported that the BC4 F1 seeds have 99.0\% recurrent parent genome after four rounds of backcrossing [60]. In the future, the analysis of genome sequencing data of all BR signaling-related factors and the examination of specific correlations between SNPs and traits in different germplasm lines can help reveal additional mechanistic details of BR and the interactions between interdependent pathways.

\section{Conclusion}

BRs play crucial regulatory roles during soybean vegetative development. Our study demonstrated that GmBZL3 plays a conserved role in the soybean BR response pathway. Our results provide a genomic map of GmBZL3 actions in soybean revealing a regulatory network that which integrates hormonal and multiple regulatory pathways for plant growth and reveals numerous molecular links between the BR signaling pathway and downstream components involved in developmental and physiological processes. In addition, the natural variation in germplasm lines identified here would provide good mutations resources for gene functional analysis in the future.

\section{Methods}

Expression profiling analysis using RNA-seq datasets

The RNA-seq data generated by Libault et al. [28] from nine different soybean tissues (Williams 82 genotype) including flowers, leaves, nodules, pods, roots, root hairs, 
seeds, shoot apical meristems and stems, was used to analyze the expression patterns of four orthologous GmBZLs. Hierarchical clustering of expression data was performed using dCHIP software [61]. The RPKM value shown presents the normalized total reads obtained in each tissue library. For salt or ABA treatments, the soybean seedling (V1 stage) roots were immersed in solutions containing $200 \mathrm{mM} \mathrm{NaCl}$ or $100 \mu \mathrm{M}$ ABA under room temperature with water as a control. For cold treatment, seedlings were kept at $4{ }^{\circ} \mathrm{C}$ with $16 \mathrm{~h}$ light $/ 8 \mathrm{~h}$ dark. For dehydration treatment, seedlings were transferred onto filter paper, and dried at room temperature with $60 \%$ humidity. First trifoliate leaves from three plants after $8 \mathrm{~h}$ treatment were harvested for RNA isolation. Tissue samples were collected from three biological replicates, and three independent experiments were repeated. Four sets of RNA-seq data with BR synthesis inhibitor treatments were used to analyze the expression patterns of GmBZL3 targets (Song et al., unpublished). Briefly, soybean seedlings at the V1 stage were treated with a high concentration BR synthesis inhibitor $(5 \mu \mathrm{M}$ propiconazole for 10 days, indicated as Pcz) or were simultaneously treated with a high concentration inhibitor and low concentration brassinolide (BL) $(5 \mu \mathrm{M}$ Pcz with $10 \mathrm{nM}$ BL for 10 days, indicated as Pcz-BL). Moreover, a high concentration of $\mathrm{BL}$ was applied in a short time after 10 days of treatment with a high concentration inhibitor $(5 \mu \mathrm{M}$ Pcz for 10 days then with $1 \mu \mathrm{M}$ BL $1 \mathrm{~h}$, indicated as Pcz-BL-1 h; $5 \mu \mathrm{M}$ Pcz for 10 days then with $1 \mu \mathrm{M}$ BL for $8 \mathrm{~h}$, indicated as Pcz-BL-8 h).

\section{Protein sequence alignments and gene duplications of GmBZLs}

Multiple sequence alignments were constructed using ClustalW2 (https://www.ebi.ac.uk/Tools/msa/clustalo/). The Ks and Ka values were extracted from the Plant Genome Duplication Database (PGDD) [62], and the values were used to calculate the approximate dates of duplication events. The date of duplication events was subsequently estimated according to the eq. $T=K s / 2 \lambda$, in which the mean synonymous substitution rate $(\lambda)$ for soybean is $6.1 \times 10^{-9}[63]$.

\section{Gene cloning, site-directed mutagenesis and genetic transformation}

The full-length open reading frame (ORF) of the GmBZL3 gene was amplified from Williams 82 with the following primer pairs: Glyma06g03700F: CACCATGAC TTCGGACGGAGCAAC and Glyma06g03700R: AGCA GGCGTCTTCCCACTTCCAAGT. Then the PCR products were cloned into the $\mathrm{pENTR}^{\mathrm{TM}} \mathrm{D} / \mathrm{TOPO}$ vector (Thermo Fisher Scientific, Waltham, MA, USA). The positive clone was fully sequenced with M13 sequencing primers. An LR gateway reaction was performed with the binary vector pEarleyGate 101 [64]. The Phusion Site-Directed Mutagenesis Kit (Thermo Scientific) was used to create the BZL3 ${ }^{P 219 L}$ mutant gene containing the Pro-219-Leu [42] mutation site with the following primers: 14-3F: GAGGATATCAAACGCTGCCCCTGTTACCC; 14-3R: AAAGGAGGGAGAGACGAGGGAAACGCAT. The GmBZL3-pEarleyGate 101 and GmBZL3 ${ }^{P 219 L}$-pEarleyGate 101 binary vectors were used for Arabidopsis transformation.

\section{Plant material, growth condition, and treatments}

We received the Arabidopsis lines bri1-5 and Ws-0 from Zhiyong Wang (Carnegie Institution for Science, Stanford, Wang et al., 2002 [10]). GmBZL3 and GmBZL3 ${ }^{P 219 L}$ were overexpressed in the Arabidopsis BR-insensitive mutant bri1-5. The Agrobacterium-mediated floral dip transformation method was employed to generate transgenic plants [65]. T3 homozygous transgenic lines were screened and used for the following phenotype analysis. Ws-0, bri1-5, overexpressed GmBZL3/bril1-5 and overexpressed GmBZL $3^{\mathrm{P} 219 \mathrm{~L}} /$ bril1-5 lines were planted and grown on $1 / 2$ solid MS media with $2 \%$ sucrose. For seedlings grown in the dark, the seeds were treated in cold for 2 days and then grown at $22^{\circ} \mathrm{C}$ for 4 days in the dark. For light grown seedlings, cold treated seeds were grown at $22{ }^{\circ} \mathrm{C}$ for 6 days under a $14 / 10 \mathrm{~h}$ light/dark photoperiod and $100 \mu \mathrm{mol} / \mathrm{m}^{2} / \mathrm{s}$ light intensity. For the analysis of GmBZL3 and $G m B Z L 3^{\mathrm{P} 219 \mathrm{~L}}$ gene expression levels in Arabidopsis transgenic plants, seedlings grown for 7 days in the light were used for RNA isolation. For BR response evaluation, cold treated seeds were grown at $22^{\circ} \mathrm{C}$ for 7 days under a $14 / 10 \mathrm{~h}$ light/dark photoperiod on $1 / 2$ solid MS media with varied $\mathrm{BL}$ concentrations $(0,1 \mathrm{nM}$, $10 \mathrm{nM}, 100 \mathrm{nM}$, and $500 \mathrm{nM}$ ).

\section{GmBZL3 antibody and genome-wide chromatin immunoprecipitation}

The peptide (FAPSVSAVPISPT) from 244 to 256 of the deduced amino acids sequence of GmBZL3 was used for antibody production in rabbits. Antibodies were produced by Pierce Company (Thermo Scientific, Huntsville, AL). ChIP assays were performed according to the published protocol [66]. Briefly, $8 \mathrm{~g}$ of soybean leaves (V3 stage) were grounded and cross-linked with $1 \%(v / v)$ formaldehyde under a vacuum for $5 \mathrm{~min}$, and then cross-linking was quenched by adding glycine to a final concentration of $0.25 \mathrm{M}$. The sample was kept under vacuum was continued for 5 more minutes. The cross-linked tissue was ground into powder in liquid nitrogen, and $5 \mathrm{~g}$ of tissue powder was used to isolate the chromatin complexes. Subsequently, polyclonal GmBZL3 antibodies were sonicated and immunoprecipitated. DNA was extracted by using a Fermentas PCR Purification kit. The 
precipitated DNA was resuspended in $50 \mu \mathrm{l}$ TE and stored at $-20^{\circ} \mathrm{C}$ for ChIP-seq analysis.

\section{ChIP sequencing and analysis}

Two independent biological replicates were used for ChIP analysis. The DNA extracted from soybean leaves without ChIP treatment was used as a negative control. ChIP sequencing library construction and sequencing were conducted by University of Missouri DNA Core Facility using a Hi-seq 2000 (Illumina, San Diego, CA). The data analysis was performed as follows: the paired end reads were aligned to the soybean genomes using Bowtie [67]. The mapping results were saved as BAM files [68]. The significantly enriched ChIP regions were identified by the peak-calling program MACS, and the results were saved as the coordinates of the identified peaks on the genome [69]. The gene-calling tool (bedtools) was then used to characterize the genes around the peak regions [70].

\section{Quantitative real-time PCR assays}

Total RNA was isolated from either soybean leaf or Arabidopsis seedlings with an RNeasy Plant mini kit (Qiagen, Cat\#:74904). On-column DNase digestion with the RNase-Free DNase set was performed to remove DNA contamination (Qiagen, Valencia, CA, Cat\#:79254). A High Capacity cDNA Reverse Transcription Kit (Thermo, USA, Cat\#4368814) was used for cDNA synthesis. The qRT-PCR and ChIP-qPCR assays were carried out using SYBR Green master mix (Thermo, USA, Cat\# K0223). The comparative $\mathrm{Ct}$ method was used to quantify the relative expression of specific genes [71]. The cyclin gene (Glyma10g263500) was selected as an internal control to normalize gene expression. All primers were designed using the Primer3 web interface (http://bioinfo.ut.ee/primer3-0.4.0/; [72]). Three biological replicates and repeated once as a technical replicate were performed in each reaction. The overexpression of the transgene was confirmed by using the primers GmBZL3-RTF(GGTC GTTTAATTGGAGGAGAAT) and (GmBZL3-RTR GATG AGGCCTATCATTTCCTG), which spanned the linking region between YFP and the transgene to exclude the possibility of Arabidopsis endogenous gene amplification. pEarly101-YFP-F (GTAAACGGCCACAAGTTCAG) and pEarly101-YFP-R(ACTTCAGGGTCAGCTTGC) were used for the gene expression level analysis in the transgenic Arabidopsis plants. The cyclin gene was chosen as an internal control by using primers as: Gm10g263500-RTF (ACCACAATGACCAACTAGA GC) and Gm10g263500-RTR (CTTCCTCTTCCCAC TTTCCTTC). The PP2A gene was chosen as ChIP-qPCR internal control. Primers of ChIP-qPCR assay are listed in Additional file 7.

\section{Additional files}

Additional file 1: Estimates of the dates for the segmental duplication events of the BZL gene pairs in soybean. (XLSX $9 \mathrm{~kb}$ )

Additional file 2: Expression patterns of GmBZLs in different tissues and under abiotic stress conditions. (A) Expression profiles of GmBZL genes in 9 tissues. The relative expression data of 9 tissues determined by RNA-seq were obtained from Libault et al.. 2010 and used to construct the expression patterns of soybean genes. Color in the heatmaps represents RPKM values of the GmBZL genes. (B-C) Transcription levels of GmBZL genes in response to abiotic stress. The soybean V1 stage seedlings were treated with $200 \mathrm{mM}$ $\mathrm{NaCl}$ or $100 \mu \mathrm{M}$ ABA for $8 \mathrm{~h}$. For cold treatment, seedlings were kept at $4{ }^{\circ} \mathrm{C}$ with light. For dehydration treatment, seedlings were transferred onto filter paper and dried at room temperature with $60 \%$ humidity. Relative gene expression levels are shown following normalization with actin transcript values. Error bars represent the standard error of the mean. The star $\left(^{*}\right)$ indicates statistically significant differences among the means $(p<0.05)$. (PPTX $88 \mathrm{~kb})$

Additional file 3: DWF4 was significantly enriched in both IP biological repeat samples compared with PP2A, but no significant different of amount between DWF4 and PP2A in negative ChIP experiment. (PPTX 67 kb)

Additional file 4: ChIP data reveal genes potentially regulated by the GmBZL3 transcription factor in soybean. (XLSX $38 \mathrm{~kb}$ )

Additional file 5: Overrepresented GO categories in GmBZL3 targets. Functional classification of GmBZL3 targets indicated that seven sets of $\mathrm{GO}$ (molecular function) terms were significantly enriched ( $p$ values $<$ 0.01). (PPTX $40 \mathrm{~kb}$ )

Additional file 6: Identification of nonsynonymous SNPs and deletions in the GmBZL3 gene from 106 soybean resequencing datasets (Valliyodan et al. 2016). Highlighted text showing SNPs and nonsynonymous mutations. (XLSX $13 \mathrm{~kb}$ )

Additional file 7: The list of primers used in $q R T-P C R$ and ChIP-qPCR. (XLSX $10 \mathrm{~kb}$ )

\section{Abbreviations}

AtMYB70: Myb domain protein 70; BAG1: BCL-2-associated athanogene 1; BC4: Back cross; bHLH: Basic helix-loop-helix; BL: Brassinolide; bri1: BRinsensitive mutant; BRs: Brassinosteroids; BXL2: Beta-xylosidase 2; ChIPchip: Chromatin-immunoprecipitation microarray; ChIP-seq: Chromatin Immunoprecipitation Sequencing; CUT1: Cuticular 1; ERD15: Early responsive to dehydration 15; EXPA1: Expansin A1; EXPA8: Expansin A8; FQR1: Flavodoxin-like quinone reductase 1; GH3: Gretchen Hagen 3; GmBZLs: BZR1-like proteins; GPRI1: GOLDEN2-like 1; GWAS: Genome-wide association study; HAT3: Homeobox-leucine zipper protein 3; HMG1: 3hydroxy-3-methylglutaryl coa reductase; JA: Jasmonate; JAZ1: Jasmonate ZIM-domain protein 1; KAB1: Potassium channel beta subunit; LBD39: Lateral organ boundaries domain protein 39; LBD41: Lateral organ boundaries domain protein 41; LOX2: Lipoxygenase 2; Mya: Million years ago; NRT1.1: Nitrate transporter 1.1; NRT1.2: Nitrate transporter 1.2; OPR2: 12oxophytodienoic acid reductases 2; ORF: Open reading frame; PCZ: Propiconazole; PGDD: Plant Genome Duplication Database; PIP1B: Plasma membrane intrinsic protein 1;2; PIP3: Plasma membrane intrinsic protein 3; PME3: Pectin methylesterase 3; qRT-PCR: Quantitative realtime PCR; RD26: Responsive to desiccation 26; RD26: Responsive to dessication 26; RPKM: Reads per kilo base; SAURs: Small auxin-up RNAs; SNPs: Single nucleotide polymorphisms; SPT: SPATULA; VTC4: Inositol monophosphatase family protein; YUCCA3: Indole-3-pyruvate monooxygenase

\section{Acknowledgements}

We are grateful to Dr. Hongwei Xue for critical reading and improvement of the manuscript.

\section{Funding}

This study was supported by the National Natural Science Foundation of China (31871540), MSMC (Missouri Soybean Merchandising Council, USA), USB (United Soybean Board. USA) and the opening research projects of national key laboratory of plant molecular genetics SIPPE. CAS. 2018. 


\section{Availability of data and materials}

All the data about the present study has been included in the table and/or figure form in the current manuscript or the supplement already. Authors are pleased to share analyzed/raw data and plant materials upon reasonable request.

\section{Authors' contributions}

WC, LS, Z-YW, BV and HTN designed the research. WC and LS performed the experiments and data analysis. BW performed ChIP-seq experiment and data analysis. Q-MY performed bioinformatics analysis and revised the manuscript. $M-Y B, Z-M Z$ and $H Y$ contributed to the experimental designing and results analysis. BV contributed to the soybean genome sequence analysis. LS and HTN wrote the manuscript draft. All authors participated in writing and approved the final manuscript.

\section{Ethics approval and consent to participate} Not applicable.

\section{Consent for publication}

Not applicable.

\section{Competing interests}

The authors declare that they have no competing interests.

\section{Publisher's Note}

Springer Nature remains neutral with regard to jurisdictional claims in published maps and institutional affiliations.

\section{Author details}

'Joint International Research Laboratory of Agriculture and Agri-Product Safety, Jiangsu Key Laboratory of Crop Genomics and Molecular Breeding, Co-Innovation Center for Modern Production Technology of Grain Crops, Yangzhou University, Yangzhou 225009, China. 'Division of Plant Sciences, University of Missouri, Columbia, MO 65211, USA. ${ }^{3}$ School of Agriculture and Biology, Shanghai Jiao Tong University, Shanghai 200240, China. ${ }^{4}$ Department of Computer Science, Informatics Institute, and Christopher S. Bond Life, Sciences Center, University of Missouri, Columbia, MO 65211, USA. ${ }^{5}$ Department of Plant Biology, Carnegie Institution for Science, Stanford, CA 94305, USA. ${ }^{6}$ Present address: Shandong University, Jinan, Shandong, China. 7 Present address: Agronomy College of Shenyang Agricultural University, Shenyang, Liaoning, China.

Received: 25 June 2018 Accepted: 7 February 2019 Published online: 22 February 2019

\section{References}

1. Li J, Chory J. Brassinosteroid actions in plants. J Exp Bot. 1999;50:332-40.

2. Clouse SD. Arabidopsis mutants reveal multiple roles for sterols in plant development. Plant Cell. 2002;14:1995-2000.

3. Ye Q, Zhu W, Li L, Zhang S, Yin Y, Ma H, et al. Brassinosteroids control male fertility by regulating the expression of key genes involved in Arabidopsis anther and pollen development. Proc Natl Acad Sci U S A. 2010;107:6100-5.

4. Zhu JY, Sae-Seaw J, Wang ZY. Brassinosteroid signalling. Development. 2013;140:1615-20.

5. Kagale S, Divi UK, Krochko JE, Keller WA, Krishna P. Brassinosteroid confers tolerance in Arabidopsis thaliana and Brassica napus to a range of abiotic stresses. Planta. 2007:225:353-64.

6. Divi UK, Krishna P. Brassinosteroids confer stress tolerance. In: Plant stress biology: from genomics goes systems biology edited by: Hirt H. Weinheim: Wiley-VCH; 2009. p. 119-35

7. Mathur J, Molnár G, Fujioka S, Takatsuto S, Sakurai A, Yokota T, et al. Transcription of the Arabidopsis CPD gene, encoding a steroidogenic cytochrome P450, is negatively controlled by brassinosteroids. Plant J. 1998;14:593-602.

8. Bancoş S, Nomura T, Sato T, Molnár G, Bishop GJ, Koncz C, et al. Regulation of transcript levels of the Arabidopsis cytochrome p450 genes involved in brassinosteroid biosynthesis. Plant Physiol. 2002;130(1):504-13.

9. Symons GM, Reid JB. Brassinosteroids do not undergo long-distance transport in pea. Implications for the regulation of endogenous brassinosteroid levels. Plant Physiol. 2004;135(4):2196-206.
10. Wang ZY, Nakano T, Gendron J, He J, Chen M, Vafeados D, et al. Nuclearlocalized BZR1 mediates brassinosteroid-induced growth and feedback suppression of brassinosteroid biosynthesis. Development Cell. 2002;2:505-13.

11. He JX, Gendron JM, Sun Y, Gampala SS, Gendron N, Sun CQ, et al. BZR1 is a transcriptional repressor with dual roles in brassinosteroid homeostasis and growth responses. Science. 2005;30:1634-8.

12. Yin $Y$, Vafeados $D$, Tao $Y$, Yoshida S, Asami T, Chory J. A new class of transcription factors mediates brassinosteroid-regulated gene expression in Arabidopsis. Cell. 2005;120:249-59.

13. Gampala S, Kim TW, He JX, Tang W, Deng Z, Bai MY, et al. An essential role for 14-3-3 proteins in brassinosteroid signal transduction in Arabidopsis. Development Cell. 2007;13:177-89.

14. He JX, Gendron JM, Yang Y, Li J, Wang ZY. The GSK3-like kinase BIN2 phosphorylates and destabilizes BZR1, a positive regulator of the brassinosteroid signaling pathway in Arabidopsis. Proc Natl Acad Sci U S A. 2002;99:10185-90

15. Yu X, Li L, Zola J, Aluru M, Ye H, Foudree A, et al. A brassinosteroid transcriptional network revealed by genome-wide identification of BESI target genes in Arabidopsis thaliana. Plant J. 2011;65:634-46.

16. Sun Y, Fan XY, Cao DM, Tang W, He K, Zhu JY, et al. Integration of brassinosteroid signal transduction with the transcription network for plant growth regulation in Arabidopsis. Development Cell. 2010;19:65-77.

17. Terakado J, Fujihara S, Goto S, Kuratani R, Suzuki Y, Yoshida S, et al. Systemic effect of a brassinosteroid on root nodule formation in soybean as revealed by the application of brassinolide and brassinazole. Soil Sci Plant Nutr. 2005:51:389-95.

18. Zurek DM, Clouse SD. Molecular cloning and characterization of a brassinosteroid-regulated gene from elongating soybean (Glycine max L.) epicotyls. Plant Physiology. 1994;104:161-70.

19. Oh MH, Romanow W, Smith R, Zamski E, Sasse J, Clouse S. Soybean BRU1 encodes a functional xyloglucan endo-transglycosylase that is highly expressed in inner epicotyl tissues during brassinosteroid-promoted elongation. Plant Cell Physiol. 1998;39:124-30.

20. Hunter WJ. Influence of Root-Applied Epibrassinolide and Carbenoxolone on the Nodulation and Growth of Soybean (Glycine max L.) Seedlings. J Agron Crop Sci. 2001;186:217-21.

21. Zhang M, Zhai Z, Tian X, Duan L, Li Z. Brassinolide alleviated the adverse effect of water deficits on photosynthesis and the antioxidant of soybean (Glycine max L.). Plant Growth Regul. 2008a;56:257-64.

22. Wang $M, X u X$, Zhang $X$, Sun $S$, Wu C, Hou W, et al. Functional Analysis of GmCPDs and Investigation of Their Roles in Flowering. PLoS One. 2015;10(3):e0118476.

23. Wang M, Sun S, Wu C, Han T, Wang Q. Isolation and characterization of the Brassinosteroid receptor gene (GmBRI1) from Glycine max. Int J Mol Sci. 2014:15:3871-88.

24. Peng S, Tao P, Xu F, Wu A, Huo W, Wang J. Functional Characterization of Soybean Glyma04g39610 as a Brassinosteroid Receptor Gene and Evolutionary Analysis of Soybean Brassinosteroid Receptors. Int J Mol Sci. 2016;17(6). https://doi.org/10.3390/ijms17060897.

25. Zhang Y, Zhang YJ, Yang BJ, Yu XX, Wang D, Zu SH, et al. Functional characterization of GmBZL2 (AtBZR1 like gene) reveals the conserved BR signaling regulation in Glycine max. Science Report. 2016;6:31134.

26. Ariyoshi Y, Itoyama H, Nakagawa ACS, Ario N, Kondo Y, Tomita Y, et al. Regulation of brassinosteroid on pod growth through cell hypertrophy in soybean (Glycine max L.) Merr. Plant Growth Regul. 2016;80:391-5.

27. Schmutz J, Cannon SB, Schlueter J, Ma J, Mitros T, Nelson W, et al. Genome sequence of the palaeopolyploid soybean. Nature. 2010;463:178-83.

28. Libault M, Farmer A, Joshi T, Takahashi K, Langley RJ, Franklin LD, et al. An integrated transcriptome atlas of the crop model Glycine max. And its use in comparative analyses in plants. Plant J. 2010;63:86-99.

29. Divi UK, Rahman T, Krishna P. Brassinosteroid-mediated stress tolerance in Arabidopsis shows interactions with abscisic acid, ethylene and salicylic acid pathways. BMC Plant Biol. 2010;10:151.

30. Goda H, Shimada Y, Asami T, Fujioka S, Yoshida S. Microarray analysis of brassinosteroid-regulated genes in Arabidopsis. Plant Physiol. 2002;130:1319-34.

31. Nemhauser JL, Mockler TC, Chory J. Interdependency of brassinosteroid and auxin signaling in Arabidopsis. PLoS Biol. 2004;2:E258.

32. Vert $G$, Walcher $\mathrm{CL}$ Chory J, Nemhauser $\mathrm{UL}$ Integration of auxin and brassinosteroid pathways by auxin response factor 2. Proc Natl Acad Sci U S A. 2008;105:9829-34.

33. Gendron JM, Haque A, Gendron N, Chang T, Asami T, Wang ZY. Chemical genetic dissection of brassinosteroid-ethylene interaction. Mol Plant. 2008;1:368-79. 
34. Zhou XY, Song $L$, Xue HW. Brassinosteroids regulate the differential growth of Arabidopsis hypocotyls through auxin signaling components IAA19 and ARF7. Mol Plant. 2013;6:887-904.

35. Thines B, Katsir L, Melotto M, Niu Y, Mandaokar A, Liu G, et al. JAZ repressor proteins are targets of the SCF(COI1) complex during jasmonate signalling. Nature. 2007:448:661-5.

36. Lozano-Durán R, Macho AP, Boutrot F, Segonzac C, Somssich IE, Zipfel C. The transcriptional regulator BZR1 mediates trade-off between plant innate immunity and growth. eLife. 2013;2:e00983.

37. Zhiponova MK, Vanhoutte I, Boudolf V, Betti C, Dhondt S, Coppens F, et al. Brassinosteroid production and signaling differentially control cell division and expansion in the leaf. New Phytol. 2013;197(2):490-502.

38. Hartwig T, Corvalan C, Best NB, Budka JS, Zhu JY, Choe S, et al. Propiconazole is a specific and accessible brassinosteroid (BR) biosynthesis inhibitor for Arabidopsis and maize. PLoS One. 2012;7(5):e36625.

39. Valliyodan B, Qiu D, Patil G, Zeng P, Huang J, Dai L, et al. Landscape of genomic diversity and trait discovery in soybean. Scientific Reports. 2016;6:23598.

40. Rechsteiner M, Rogers SW. PEST sequences and regulation by proteolysis. Trends Biochem Sci. 1996;21:267-71.

41. Bai MY, Zhang LY, Gampala SS, Zhu SW, Song WY, Chong K, et al. Functions of OsBZR1 and 14-3-3 proteins in Brassinosteroid signaling in Rice. Proc Natl Acad Sci U S A. 2007;104:13839-44.

42. Tang W, Yuan M, Wang R, Yang Y, Wang C, Oses-Prieto JA, et al. PP2A activates brassinosteroid-responsive gene expression and plant growth by dephosphorylating BZR1. Nat Cell Biol. 2011;13:124-31.

43. Peng P, Li J. Brassinosteroid signal transduction: a mix of conservation and novelty. J Plant Growth Regul. 2003;22:298-312.

44. Hao J, Yin Y, Fei SZ. Brassinosteroid signaling network: implications on yield and stress tolerance. Plant Cell Rep. 2013;32:1017-30.

45. Sairam RK. Effects of homobrassinolide application on plant metabolism and grain yield under irrigated and moisture-stress conditions of two wheat varieties. Plant Growth Regul. 1994;14:173-81.

46. Sahni S, Prasad BD, Liu Q, Grbic V, Sharpe A, Singh SP, et al. Overexpression of the brassinosteroid biosynthetic gene DWF4 in Brassica napus simultaneously increases seed yield and stress tolerance. Sci Rep. 2016;6:28298.

47. Feng Y, Yin Y, Fei S. Down-regulation of BdBRI1, a putative brassinosteroid receptor gene produces a dwarf phenotype with enhanced drought tolerance in Brachypodium distachyon. Plant Sci. 2015;234:163-73.

48. Gui J, Zheng S, Liu C, Shen J, Li J, Li L. OsREM4.1 interacts with OsSERK1 to coordinate the interlinking between abscisic acid and brassinosteroid signaling in rice. Development Cell. 2016;38:201-13.

49. Nolan TM, Brennan B, Yang M, Chen J, Zhang M, Li Z, et al. Selective autophagy of BES1 mediated by DSK2 balances plant growth and survival. Development Cell. 2017;41:33-46.

50. Ye H, Liu S, Tang B, Chen J, Xie Z, Nolan TM, et al. RD26 mediates crosstalk between drought and brassinosteroid signalling pathways. Nature Communication. 2017;24(8):14573.

51. Kariola T, Brader G, Helenius E, Li J, Heino P, Palva ET. EARLY RESPONSIVE TO DEHYDRATION 15. A negative regulator of abscisic acid responses in Arabidopsis. Plant Physiol. 2006;142:1559-73.

52. Li J, Nagpal P, Vitart V, McMorris TC, Chory J. A role for brassinosteroids in light-dependent development of Arabidopsis. Science. 1996;272:398-401.

53. Homrich MS, Wiebke-Strohm B, Weber RL, Bodanese-Zanettini MH. Soybean genetic transformation: a valuable tool for the functional study of genes and the production of agronomically improved plants. Genet Mol Biol. 2012;35:998-1010.

54. Indrasumunar A, Searle I, Lin MH, Kereszt A, Men A, Carroll BJ, et al. Nodulation factor receptor kinase 1a controls nodule organ number in soybean (Glycine max L. Merr). Plant J. 2011;65:39-50.

55. Kasai M, Kanazawa A. RNA silencing as a tool to uncover gene function and engineer novel traits in soybean. Breed Sci. 2012;61:468-79.

56. Yang L, Ji W, Zhu YM, Gao P, Li Y, Cai H, et al. GsCBRLK, a calcium/ calmodulin-binding receptor-like kinase, is a positive regulator of plant tolerance to salt and ABA stress. J Exp Bot. 2010;61:2519-33.

57. Hyten DL, Cannon SB, Song Q, Weeks N, Fickus EW, Shoemaker RC, et al. High-throughput SNP discovery through deep resequencing of a reduced representation library to anchor and orient scaffolds in the soybean whole genome sequence. BMC Genomics. 2010;11:38.

58. Lam HM, Xu X, Liu X, Chen W, Yang G, Wong FL, et al. Resequencing of 31 wild and cultivated soybean genomes identifies patterns of genetic diversity and selection. Nat Genet. 2010;42:1053-9.
59. Zhou Z, Jiang Y, Wang Z, Gou Z, Lyu J, Li W, et al. Resequencing 302 wild and cultivated accessions identifies genes related to domestication and improvement in soybean. Nat Biotechnol. 2015;33:408-14.

60. Hospital F. Marker-assisted breeding. In: H.J. Newbury (ed.). Plant molecular breeding, vol. 2003. Oxford and Boca Raton: Blackwell publishing and CRC press; 2003. p. 30-59.

61. Li C, Wong WH. Model-based analysis of oligonucleotide arrays: expression index computation and outlier detection. Proc Natl Acad Sci U S A. 2001;98:31-6.

62. Lee $T H$, Tang H, Wang X, Paterson AH. PGDD: a database of gene and genome duplication in plants. Nucleic Acids Res. 2013. https://doi.org/10. 1093/nar/gks1104.

63. Lynch M, Conery JS. The evolutionary fate and consequences of duplicate genes. Science. 2000;290:1151-5.

64. Earley KW, Haag JR, Pontes O, Opper K, Juehne T, Song K, et al. Gatewaycompatible vectors for plant functional genomics and proteomics. Plant J. 2006;45:616-29.

65. Clough SJ, Bent AF. Floral dip: a simplified method for Agrobacteriummediated transformation of Arabidopsis thaliana. Plant J. 1998;16:735-43.

66. Zhu JY, Sun Y, Wang ZY. Genome-wide identification of transcription factor-binding sites in plants using chromatin immunoprecipitation followed by microarray (ChIP-chip) or sequencing (ChIP-seq). Methods Mol Biol. 2012;876:173-88.

67. Langmead B, Salzberg S. Fast gapped-read alignment with bowtie 2. Nat Methods. 2012;9:357-9.

68. Li H, Handsaker B, Wysoker A, Fennell T, Ruan J, Homer N, et al. 1000 genome project data processing subgroup. The sequence alignment/map (SAM) format and SAMtools. Bioinformatics. 2009;25:2078-9.

69. Zhang Y, Liu T, Meyer CA, Eeckhoute J, Johnson DS, Bernstein BE, et al. Model-based analysis of ChIP-Seq (MACS). Genome Biol. 2008b;9(9):R137. https://doi.org/10.1186/gb-2008-9-9-r137.

70. Maurano MT, Humbert R, Rynes E, Thurman RE, Thurman RE, Haugen E, et al. Systematic localization of common disease-associated variation in regulatory DNA. Science. 2012;337:6099.

71. Livak KJ, Schmittgen TD. Analysis of relative gene expression data using real-time quantitative PCR and the 2(-Delta Delta C(T)) method. Methods. 2001:25:402-8.

72. Rozen S, Skaletsky H. Primer3 on the WWW for general users and for biologist programmers. Methods Mol Biol. 2000;132:365-86.
Ready to submit your research? Choose BMC and benefit from:

- fast, convenient online submission

- thorough peer review by experienced researchers in your field

- rapid publication on acceptance

- support for research data, including large and complex data types

- gold Open Access which fosters wider collaboration and increased citations

- maximum visibility for your research: over $100 \mathrm{M}$ website views per year

At BMC, research is always in progress.

Learn more biomedcentral.com/submissions 